\title{
Compact quasi-monoenergetic photon sources from laser-plasma accelerators for nuclear detection and characterization
}

Cameron G.R. Geddes ${ }^{\mathrm{a}, *}$, Sergey Rykovanov ${ }^{\mathrm{a}, \mathrm{b}}$, Nicholas H. Matlis ${ }^{\mathrm{a}}$, Sven Steinke ${ }^{\mathrm{a}}$, J.-L. Vay ${ }^{a}$, Eric H. Esarey ${ }^{a}$, Bernhard Ludewigt ${ }^{a}$, Kei Nakamura ${ }^{\text {a }}$, Brian J. Quiter ${ }^{\text {a }}$, Carl B. Schroeder ${ }^{a}$, Csaba Toth ${ }^{a}$, Wim P. Leemans ${ }^{\text {a,c }}$

${ }^{a}$ Lawrence Berkeley National Laboratory, 1 Cyclotron Road, Berkeley CA 94720

${ }^{b}$ now at: Helmholtz Institute Jena, ${ }^{c}$ also at: Department of Physics, U.C. Berkeley

\section{Abstract}

Near-monoenergetic photon sources at $\mathrm{MeV}$ energies offer improved sensitivity at greatly reduced dose for active interrogation, and new capabilities in treaty verification, nondestructive assay of spent nuclear fuel and emergency response. Thomson (also referred to as Compton) scattering sources are an established method to produce appropriate photon beams. Applications are however restricted by the size of the required high-energy electron linac, scattering (photon production) system, and shielding for disposal of the high energy electron beam. Laser-plasma accelerators (LPAs) produce GeV electron beams in centimeters, using the plasma wave driven by the radiation pressure of an intense laser. Recent LPA experiments are presented which have greatly improved beam quality and efficiency, rendering them appropriate for compact high-quality photon sources based on Thomson scattering. Designs for $\mathrm{MeV}$ photon sources utilizing the unique properties of LPAs are presented. It is shown that control of the scattering laser, including plasma guiding, can increase photon production efficiency. This reduces scattering laser size and/or electron beam current requirements to scale compatible with the LPA. Lastly, the plasma structure can decelerate the electron beam after photon production, reducing the size of shielding required for beam disposal. Together, these techniques provide a path to a compact photon source system.

(C) 2014 The Authors. Published by Elsevier B.V.

Selection and peer-review under responsibility of the Organizing Committee of CAARI 2014.

Keywords: Nonproliferation, Homeland Security, Active Interrogation, Monoenergetic Photon Source, Laser Plasma Accelerator, Wakefield

\section{Introduction}

Near-monoenergetic photon sources (MPSs) at 1-10 MeV energies offer improved sensitivity at greatly reduced dose for detection and characterization of nuclear materials. Many of the issues with current broad-band photon sources, including unnecessary dose that can interfere with the signatures to be detected and/or restrict operations [1], can be resolved using MPS capabilities to select energy, energy spread, flux, and pulse structure to deliver only the photons needed. MPS applications have however been limited by the sizes of the required high-energy electron accelerator, scattering laser, and electron beam dump. While nuclear transitions can be a source of very narrow line width photons, they are limited in selection of energies, intensity and portability. To enable new applications outside of fixed facilities, compact tunable MPSs are desired with narrow divergence to allow high spatial resolution and reduce need for collimation.

* Corresponding author. Tel.: 510-495-2923.

E-mail address: cgrgeddes@1bl.gov 
Thomson scattering of a laser from an electron beam is a well-established, tunable and narrow divergence MPS (also referred to as Compton-Scattering or Inverse Compton-Scattering) whose application is currently limited by the need for high energy electron linacs, which are large fixed facilities using conventional technology. Current $\mathrm{MeV}$ sources include HIGS at Duke [2] and TREX/MEGA-Ray at LLNL [3]. A new generation of sources is under construction including the Extreme Light Infrastructure project in Romania [4], a project at the Japan Atomic Energy Agency [5], and proposed facilities including those at FNAL [6], SLAC [7], and BNL [8]. More compact accelerators at electron energies of 200 to $600 \mathrm{MeV}$, suitable for application needs of 1-9 MeV photons (and to > 15 $\mathrm{MeV}$ if desired), are now possible using $\mathrm{cm}$-scale laser-plasma accelerators (LPAs). Initial experiments have demonstrated Thomson scattering photon production from LPAs [9] by placing a foil to back-reflect the drive laser onto the electron beam [10], or a femtosecond laser split from the LPA driver [11,12], but so far have resulted in broad bandwidth photon beams. LPA electron beam quality and scattering laser control must be improved to generate narrow bandwidth sources.

Heavy shielding is conventionally required due to the high electron beam energy in Thomson MPSs. The low photon production cross section of Thomson scattering has also hitherto required either high electron current (and thus heavier shielding) or very large scattering lasers. Techniques to reduce shielding needs and scattering laser size, which can dominate overall system size and weight as the electron accelerator is made smaller, are hence important.

In this proceedings we show how a compact high flux Thomson scattering based MPS can be achieved by integrating a high quality LPA with techniques for scattering efficiency and for reduced shielding needs. MPS flux and bandwidth parameter ranges suitable for applications are reviewed in Section 2, and the implications of these for Thomson source design in Section 3. Development of high quality LPAs to provide the required electron source is described in Section 4. Techniques to achieve high photon flux with low bandwidth while reducing electron current and scattering laser size are presented in Section 5. Using the LPA to decelerate the electron beam to low energy after photon production can greatly reduce shielding requirements, as discussed in section 6.

\section{Monoenergetic photon applications}

Experiments on existing fixed-facility sources, as well as simulations, have characterized the MPS beam photon flux, bandwidth, and repetition rates required to improve detection and/or characterization of nuclear material, as well as for nuclear physics studies. Modalities of interest for nuclear studies include radiography, photofission, and Nuclear Resonance Fluorescence (NRF). Photon energy, bandwidth, flux, and pulse structure are primary criteria.

For Radiography, selecting the energy of the photon beam can minimize dose, while using two or more energies can improve $\mathrm{Z}$ discrimination [13]. Because radiography cross sections vary gradually at $\mathrm{MeV}$ energies, relatively broad bandwidth at the $10-25 \%$ level is sufficient. For example scanning a $2.4 \mathrm{~m}$ high container at $80 \mathrm{~cm} / \mathrm{s}$ with $1 \mathrm{~cm}$ resolution and penetrating $20 \mathrm{~cm}$ of steel (cf. ANSI N42.46) would indicate use of order $10^{6}$ photons/shot at rates $\geqslant 20 \mathrm{kHz}$, while penetration of areas $40 \mathrm{~cm}$ thick would require of order $10^{8}$ photons/shot. In this case, the repetition rate lower limit is set by the number of pixels which must be resolved. Milliradian beam divergence would allow the desired $1 \mathrm{~cm}$ resolution. To penetrate a given target thicknesses using doses approaching the lowest obtainable values, a first shot at each position can be taken with low flux, and the measured transmission used to determine attenuation for further shots at higher flux. Narrow bandwidth sources at the $10 \%$ level near $9 \mathrm{MeV}$, and at similar fluxes, also improve photofission-based interrogation and characterization by suppressing extraneous signals that can be generated with broader bandwidth or higher energy sources [14]. NRF methods, at isotope specific energies near $2 \mathrm{MeV}$, offer powerful isotope-specific identification for verification of absence of a substance [15], NDA of fuel [5], and the full range of applications. While the resonance line bandwidth is very narrow $\left(\sim 10^{-6}\right.$ at room temperature), numerous studies (e.g. [5,15]) show that MPS bandwidth at the percent level FWHM (full width at half maximum) combined with control of photon energy allows isolation of the NRF line. Typical fluxes are in the range of $10^{7} \mathrm{ph} / \mathrm{shot}$ at $\geq \mathrm{kHz}$ repetition rates. In this case, the repetition rate lower limit is set by detector considerations. Sub-percent bandwidths improve resolution and can allow use of simple calorimetric detectors. 


\section{Compact monoenergetic photon system requirements}

The parameters of a MPS in the required range of $10^{6}-10^{8}$ photons/shot can be derived from the Thomson scattering equations for energy spreads of $10-25 \%$ at energies of 2-9 MeV (radiography/photofission) or energy spreads of a few percent at energies near $2 \mathrm{MeV}$ (NRF). These are well understood and verified, to the level that scattering is used as an accelerator diagnostic on conventional machines such as the ALS linac at LBNL [16], HIGS at Duke [2], and Helmholtz-Zentrum Dresden-Rossendorf [17]. LPA-specific designs presented in detail in [18] are summarized here. Scattering near 180 degrees between the electron and photon beams (backscatter) is chosen for

\section{.} . peak photon energy. $\mathrm{MeV}$ photons are produced by upshifting of the scattering laser due to the relativistic motion of the electron beam. Available high power scattering lasers typically have $\mathrm{E}_{\mathrm{L}} \sim 1.5 \mathrm{eV}(0.8-1 \mu \mathrm{m})$ requiring a highenergy electron accelerator to produce $\mathrm{MeV}$ photons. Electrons near $200 \mathrm{MeV}$ produce $\sim 1 \mathrm{MeV}$ photons, and electrons near $600 \mathrm{MeV}$ produce $\sim 9 \mathrm{MeV}$ photons. Using conventional technology, such accelerators and the associated beam manipulation lines are large fixed facilities. Frequency up-conversion can double scattered energy to $2-18 \mathrm{MeV}$ for the same electron energies, but use of very high frequency lasers is limited by the scattering cross section issues below. Scattered photons are emitted into an angular range $\sim 1 / \gamma \sim 1 /\left(2 * \mathrm{E}_{\mathrm{e}}[\mathrm{MeV}]\right)$ around the electron beam direction and within that angular range display an energy-angle correlation resulting in a bow-like spectral intensity distribution (Fig. 1) which must be collimated to produce narrow bandwidth (limited by the on-axis bandwidth). The on-axis source bandwidth convolves electron beam energy and angular spread. The scattering laser can also contribute to this bandwidth via nonlinear broadening at high intensity, via its laser bandwidth or via multiple scattering. Limiting these effects typically requires operation of the scattering laser at low intensity $\left(\leq 10^{17}\right.$ $\mathrm{W} / \mathrm{cm}^{2}$ ) and with long pulse lengths ( $\geq 0.5 \mathrm{ps}$ ).
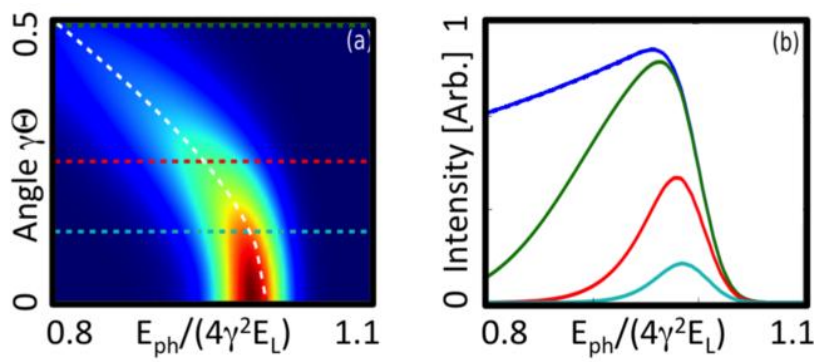

Fig. 1. A typical Thomson scattering photon spectrum, showing (a) intensity (colorscale) as a function of normalized energy and scattering angle; (b) spectra integrated over different collimator angles. The colors correspond to the collimations shown by the respective dashed lines in (a), illustrating that narrow collimation is required to achieve low bandwidth, down to the limit of the on axis bandwidth beyond which tighter collimation affects only intensity.

Due to the low scattering cross section and the need to keep scattering laser intensity low, very few (typically $<10^{-10}$ ) of the laser photons are scattered, and even scattering one photon from each electron is challenging. Lengthening the scattering laser pulse to increase yield is conventionally inefficient because it also requires increasing the laser spot size so that the laser remains focused over its pulse length. This results in wasted scattering laser energy since the laser diameter then greatly exceeds that of the electron bunch. The scattered photon yield then scales only as the square root of the laser energy. Fixed facility MPSs for this reason typically use modest laser energies and recirculate the (mostly unused) scattering laser to intersect with many electron bunches, from each of which much less than one photon is scattered per electron. This however increases the required electron current to produce a given number of photons, and hence increases both accelerator power and shielding, which is problematic in weight for transportable or small laboratory applications. In these cases, scattering efficiently at $\gtrsim 1$ photon/electron can be important.

Tradeoffs and needs for development are illustrated by source parameters relevant to photofission and NRF, each for $\sim 1$ photon per electron. For photofission, a $650 \mathrm{MeV}$ electron beam can be used to achieve $9 \mathrm{MeV}$ photon energy. Photon bandwidth of $10 \%$ FWHM indicates electron energy spread at or below $3 \%$ and divergence $\gamma \Theta \leq 0.4$, or $\sim 0.3 \mathrm{mrad}$. A $40 \mathrm{~J}$ scattering laser would produce $\sim 1$ photon/electron in this bandwidth, or $10^{8}$ photons for 
realistic bunch charges. For $25 \%$ bandwidth these parameters are relaxed, with divergence of $\sim 0.8 \mathrm{mrad}$ at 650 $\mathrm{MeV}$ being acceptable, or $1 \mathrm{mrad}$ at $450 \mathrm{MeV}$ if a frequency doubled scattering laser were used. For NRF, a 270 $\mathrm{MeV}$ electron beam can be used to produce $1.7 \mathrm{MeV}$ photons. Photon bandwidth of $2.5 \%$ FWHM (1\% RMS) requires electron beam energy spread at or below $1 \%$ and divergence of $\leq 0.1 \mathrm{mrad}$. Tighter collimation and lower laser intensity are also required, such that the same $40 \mathrm{~J}$ laser would produce 0.1 photon/electron in this bandwidth, or $10^{7}$ photons for realistic bunch charge. Details and source design tradeoffs are presented in [18]. The required very high scattering laser energy would not be realistic in power or laser size. Additional size constraint comes from the heavy shielding required for the high-energy electron beam. If the constraint on the scattering laser were relaxed by scattering fewer photons per electron and using higher electron current, shielding requirements as well as accelerator power consumption would be worsened. A solution to this tradeoff is important for a compact source.

The photon source criteria outlined indicate three important elements for a photon sources to achieve the desired photon fluxes per shot and bandwidth in a transportable, field or small laboratory format. The next sections address these in turn: a compact, high quality electron accelerator at 200-700 MeV energies (Section 4); techniques for increasing scattered photon yield for a given laser energy and electron current (Section 5); and reduction of radiation production from the high energy electron beam (Section 6). Operation at $\geq \mathrm{kHz}$ repetition rates is then required to realize the target time-averaged photon fluxes of $10^{10}-10^{12}$ photons per second. As described above such rates are in any case typically desired for resolution or detector reasons. Development of robust and efficient Joule-class short pulse (femtosecond to picosecond) lasers at $\mathrm{kHz}$ or greater repetition rates is required [19], which is a subject of concentrated effort driven by potential high energy physics applications of LPAs [20].

\section{Compact high quality electron sources using laser plasma accelerators}

LPAs produce electron energies suitable for Thomson $\mathrm{MeV}$ photon sources, $200-1000 \mathrm{MeV}$, in $\leqslant 3 \mathrm{~cm}$ plasmas (Review, [21]). System length including laser focusing optics for the LPA and scattering of $\sim 1-2 \mathrm{~m}$ is realistic (excluding the length of the laser driver). Such a package is small enough to allow rotation for rastering of a future photon source across a target. Accelerating fields orders of magnitude greater than conventional machines are achieved using a space-charge wave driven in a low density plasma by the radiation pressure of an intense laser to accelerate particles. The resonance condition for driving the wave results in predictable scaling for beam and laser energies [21]. For example [22], the drive laser for $200 \mathrm{MeV}$ electrons should require of order $10 \mathrm{TW}$ of power, 0.4 $\mathrm{J}$ energy, $40 \mathrm{fs}$ duration, and focusing to 10 micron spot sizes. These parameters are quite different from the Thomson scattering laser, requiring independent laser systems.

Over the past decade, quasi-monoenergetic beams were demonstrated at $86 \mathrm{MeV}$ [23], followed by $1000 \mathrm{MeV}$ [24] energies with few percent energy spreads and charges of $\sim 10^{8}$ electrons. In the past, required laser powers were significantly above those indicated by theory and too high for mobile application, and beam quality was insufficient to generate narrow energy spread photon beams via Thomson scattering. With LPAs as with conventional linacs, improved performance requires control of the accelerator structure and of particle injection into it.

Recent experiments [25] show that detailed laser phase-front control via a deformable mirror results in high quality laser focal spots over the full focal depth of the laser, which is not typical of TW-class lasers. This improved the propagation of the laser through the plasma. Visible transverse filamentation was eliminated, producing a collimated structure. This extended the acceleration distance resulting in energies up to $250 \mathrm{MeV}$ from the same laser which in the past was limited to $86 \mathrm{MeV}$. Using a fraction of that laser, $0.4 \mathrm{~J}$ at $10 \mathrm{TW}$, acceleration to 200 $\mathrm{MeV}$ was achieved (Fig.2). This beam energy is suitable for NRF and low energy radiography MPSs near 1-2 MeV. The laser energy used is approximately $1 / 3$ of that in previous experiments at similar electron energy and is close to theoretical scalings for efficient LPAs. $10 \mathrm{TW}$-class lasers at $10 \mathrm{~Hz}$ are now commercially available as mobile, selfcontained trailers (as noted above, $\mathrm{kHz}$ development is required). However these beams, which resulted from 'self injection' of background plasma electrons at very high wave amplitude, had energy spreads of several percent, too high for narrow bandwidth photon sources.

By controlling injection into the LPA structure using colliding laser pulses [26,27] energy spread was reduced and beam performance could be tuned. Energy spreads down to the instrument limit of the electron spectrometer at $<1.4 \%$ FWHM were produced together with divergences of $1.5 \mathrm{mrad}$ FWHM using the $10 \mathrm{TW}$ laser driver (Fig. 2) 
[28]. Energy tuning over a factor of two in energy, from $80 \mathrm{MeV}$ to above $160 \mathrm{MeV}$, was achieved. This would correspond to tuning of photon energy, in a Thomson source, by four-fold. Such tuning would allow, for example, scanning over NRF resonances or two-energy radiography. The energy spread demonstrated is consistent with photon sources of $\leq 3 \%$ FWHM energy spread, or $\leq 1 \%$ RMS, relevant to NRF.

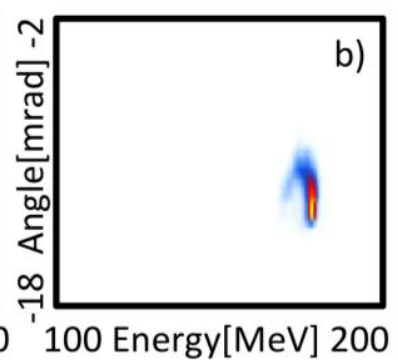

Fig. 2. LPA experiments produce high quality electron beams: colliding pulse injection (a) or self injection at $200-500 \mathrm{MeV}$ (b,c).

Related experiments demonstrated few-percent energy spread bunches at $500-1000 \mathrm{MeV}$ with few percent energy spread using 30-40 TW class lasers (Fig.2). In this case controlled guiding with a preformed plasma channel was used to further extend the laser propagation distance in the plasma to efficiently produce higher energies [24,29]. These experiments also showed that LPA emittance, a measure of focusability, matches that of high quality conventional accelerators at $0.1 \mu \mathrm{m}$ and is consistent with matched propagation in the focusing structure for the observed divergences of $1.2 \mathrm{mrad}$ RMS [29]. Beam energies are suitable to produce photons up to $9 \mathrm{MeV}$, e.g. for photofission or for $\mathrm{Z}$ discrimination in radiography. Energies up to $4 \mathrm{GeV}$ have been demonstrated in experiments on larger plasmas and lasers [30]. While LPA energy spreads are broader than state-of-the-art conventional linacs, they are sufficient for Thomson sources at 3-10\% FWHM bandwidth. Recent experiments indicate sub-percent slice energy spread in LPAs [31], which with removal of correlation could enable sub-percent photon bandwidth sources.
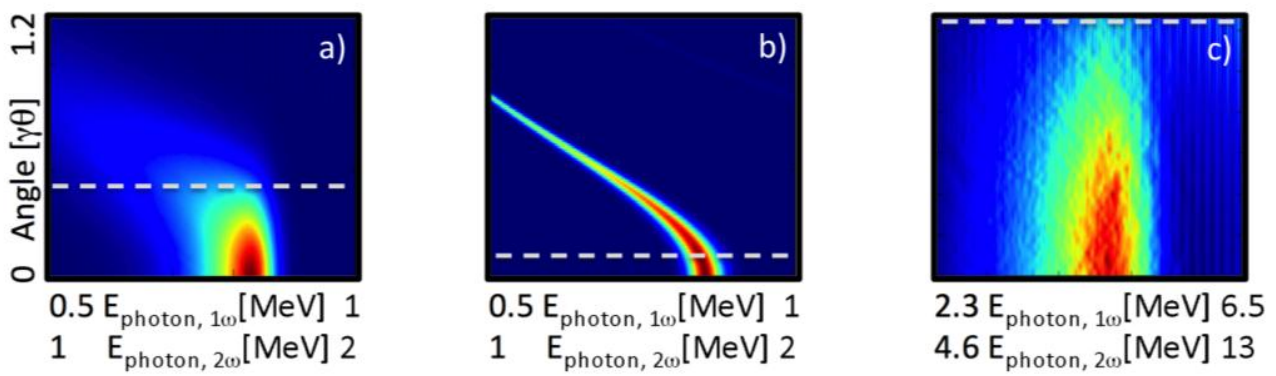

Fig. 3. Photon spectra simulated for the electron beams of high quality LPAs as shown in Figure 2, and an independent scatter laser. (a) $200 \mathrm{MeV}$ electron beams produce photon energy of $0.85 \mathrm{MeV}$ with a scattering laser of $1 \omega(800 \mathrm{~nm})$, or $1.7 \mathrm{MeV}$ with $2 \omega(400 \mathrm{~nm})$, both in $10 \%$ FWHM bandwidth set by beam divergence. (b) Few percent bandwidth could be produced by expanding the same electron beam to reduce its divergence. (c) $460 \mathrm{MeV}$ electron beams produce $4.7 \mathrm{MeV}$ or $9 \mathrm{MeV}$ photons using 800 or $400 \mathrm{~nm}$ scattering lasers respectively, at bandwidth of $25 \%$ FWHM set by divergence. As above, beam expansion can reduce bandwidth. Collimator angles for each beam are illustrated with dashed lines.

The beam quality demonstrated indicates LPAs are suitable to drive high quality MPSs, and that this can be done using Joule-class laser drivers. Examples of photon spectra calculated for the electron beams of Fig. 2 are shown in Fig. 3. Electron divergence dominates the bandwidth for scattering at the accelerator exit, but refocusing the electron beam to a larger spot size removes this limit and allows few percent FWHM photon bandwidth limited by electron energy spread. Several compact refocusing techniques are feasible [18]. Simulations indicate that energy spreads below $1 \%$ are achievable, and experiments show that slice energy spread can already be below $1 \%$ [31], providing a 
path to sub-1\% bandwidth MPSs. To control overall source size, techniques are next needed to enable high photon fluxes using for the scattering laser similar joule-class laser energies to those used for the LPA.

\section{High photon yield with reduced electron current}

Comparing to the equivalent quantity for $1 \mu \mathrm{m}$ laser light, LPA electron beam emittance is three orders of magnitude smaller, and compensating this disparity is the key to obtaining efficient photon production. For interaction in vacuum, the emittance disparity means the electron beam will stay collimated over much longer distances than a laser pulse of equivalent spot size. For the low intensities typically required to limit nonlinear contributions to photon bandwidth, interaction distances at $\mathrm{cm}$-scale are required to achieve $\sim 1$ photon per electron (as desired to obtain a given photon flux with minimal electron current and hence shielding). Focusing of the electron beam to spot sizes of $\sim 1 \mu \mathrm{m}$ RMS is sufficient for this distance as well as to meet requirements for $1 \%$ level photon bandwidth. Typical laser spot sizes to obtain these focal depths are in contrast $\sim 15 \mu \mathrm{m}$ for the examples given in Section 3, with all of the laser energy outside the electron beam radius being wasted. This is what drives the requirement for tens of joules of scattering laser energy. Since so few of the laser photons are scattered due to the low scattering cross section, there is no gain from increasing the electron beam spot size. Realizing high yield with lower scattering laser energies then requires that either the laser focal depth must be increased while keeping its radius small, or that the required interaction length must be shortened.

The scattering laser focal depth can be extended by using a plasma channel. This technique has been used successfully in LPA experiments to guide the LPA drive laser over > 10 focal depths at guided spot sizes as small as $3 \mu \mathrm{m}$ RMS [32]. These experiments use parabolic plasma density profiles with minimum density on axis, which forms a guiding fiber for the laser pulse which can withstand intensities beyond those required for Thomson scattering. Returning to the examples given in Section 2, guiding the laser pulse even with a relatively relaxed waveguide at 6 micron RMS spotsize would reduce the scattering laser energy from $40 \mathrm{~J}$ to $5 \mathrm{~J}$ at the same photon yield. A $3 \mu \mathrm{m}$ spotsize would allow energies approaching $1 \mathrm{~J}$. For very narrow bandwidth photon sources, it may be desirable to use a hollow plasma channel. This removes focusing forces on the electron beam while preserving laser focusing. Such channels are also of interest for high energy physics applications of LPAs where they can be used to control electron beam emittance [33]. Alternatively, increasing scattering laser intensity allows shorter interaction lengths while maintaining photon yield. Nonlinear detuning of scattered photon frequency has limited this approach: the high intensity peak of the laser profile scatters a different frequency than the low intensity wings. This nonlinear broadening can be compensated by designing a laser pulse with time-dependent frequency 'chirp' such that the change in frequency compensates for the nonlinear frequency shift at each time slice within the pulse. Such pulses are detailed in [34,35] and require controlled, broad bandwidth and chirp in the scattering laser. These approaches produce high fluxes $\geq 1$ photon/electron, reducing electron current, while using laser energy which is comparable to the LPA drive laser energy such to mitigate both scattering laser and shielding contributions to MPS size.

\section{Compact electron beam disposal via plasma deceleration}

To reduce radiation from the high-energy electron beam, which in conventional Thomson MPSs requires large and heavy shielding that severely limits transportability, the LPA can be used to decelerate the beam after photon production [18]. As in a conventional accelerator structure, LPAs have an accelerating and focusing phase, and an adjacent decelerating and focusing phase. The very high gradient of the LPA makes it practical to use the later to decelerate the beam since doing so would add only of order a centimeter to system length.

Because the LPA structure travels at the group velocity of the laser driver, the beam naturally slips forward in phase as it propagates. The result is that the beam moves from the accelerating to the decelerating phase after the laser has propagated a distance known as the 'dephasing length.' In typical experiments, acceleration is terminated at this length to maximize energy gain. Extending the plasma to approximately twice this length, the beam is subsequently decelerated back to low energy all in the same centimeter-scale structure. Simulations indicate deceleration of $90 \%$ or more is achievable using this simple method (Fig. 4). Because the deceleration is due to the plasma collective fields, not scattering, no significant high energy radiation is produced. Techniques to further 
increase deceleration efficiency are under development including tapering of the density, use of separate structures, or passive plasma sections.

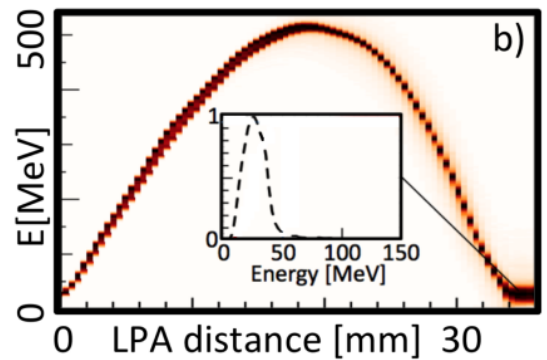

Fig. 4. (a) Snapshot of the accelerating ( $\mathrm{z}$ ) and focusing (x) fields of an LPA plasma wave. With appropriate phasing of the injection of the electron beam (green), approximately half of a period is available for simultaneous guiding and acceleration, immediately followed by simultaneous guiding and deceleration of the electron beam. (b) Energy profile of the electron beam versus propagated distance in the plasma channel show that controlled deceleration can bring the beam down to $\sim 10 \%$ of initial energy (inset: distribution), greatly reducing shielding requirements. Photons would be produced at the center of the structure where energy is nearly constant (distance of $\sim 18 \mathrm{~mm}$ in panel $b$ ).

Scattering and photon production would be conducted in the center region where the beam changes energy only slowly with distance. Simulations similar to those for Figure 3 show that scattering in this manner can produce $\sim 13 \%$ FWHM bandwidth photon sources, limited by the transverse angular distribution of the electrons in the strong focusing force of the LPA. As noted above and detailed in [18], several methods for compact electron refocusing to reduce this bandwidth are available, including tapered plasmas or plasma lenses and these can reduce divergence to allow bandwidths at or below the $1 \%$ level while maintaining compactness and compatibility with deceleration as well as guiding of the scattering laser for efficient scattering. The longitudinal and transverse momentum spreads induced by scattering are small (this is required to maintain a high quality photon source) and as a result will not significantly affect deceleration.

\section{Conclusion}

Thomson scattering based photon sources offer nearly monoenergetic, tightly collimated beams at $\mathrm{MeV}$ energies relevant to nuclear applications. Laser-plasma based techniques offer a path to reduce the size of such sources to transportable scale by addressing the size-limiting components. These includes the high-energy electron accelerator itself, where recently demonstrated cm-scale LPA performance is in the range needed for photon sources at the $10 \%$ energy spread level, or at the $\leq 3 \%$ level with refocusing of the electron beam. Sub-1\% energy spreads are realistic. With smaller accelerators, the size of other system components must be considered. Plasma channel guiding, or potentially controlled laser chirp, can resolve issues with scattering which currently require either high electron current (increasing shielding size) or large scattering lasers to achieve high photon flux. After scattering, the LPA can be used decelerate the electron beam to low energies further reducing the need for shielding. The combination of all of these elements will be important to MPS operability and to limiting extraneous radiation which could confuse detectors. Realizing the advantages of LPAs for field applications additionally requires compact, durable lasers at $\geqslant \mathrm{kHz}$ repetition rates.

\section{Acknowledgements}

This work was supported by the U.S. Dept. of Energy National Nuclear Security administration Defense Nuclear Nonproliferation R\&D (NA-22), and by the Office of Science Office of High Energy Physics, under Contract No. DE-AC02-05CH11231. The simulations used the computational facilities at the National Energy Research Scientific Computing Center, a DOE Office of Science User Facility supported by the Office of Science of the U.S. Department of Energy under Contract No. DE-AC02-05CH11231. We would like to acknowledge fruitful 
discussions with J. van Tilborg, M. Zolotorev, C. Benedetti, M. Chen, S.S. Bulanov, E. Cormier-Michel, and D. Bruhwiler and F. Rossi.

\section{References -}

[1] R. C. Runkle, D.L. Chichester, S.J. Thomson, "Rattling nucleons: New developments in active interrogation of special nuclear material," Nucl. Inst. Meth. Phys. Res. A. 663 75-95 (2012).

[2] H.R. Weller, M. W. Ahmed, H. Gao, W. Tornow, Y. K. Wu, M. Gai, R. Miskimen, "Research opportunities at the upgraded HIgS facility," Proc. Part. Nucl. Phys. 62 (2009) 257.

[3] F. Albert, S. G. Anderson, D. J. Gibson, C. A. Hagmann, M. S. Johnson, M. Messerly, V. Semenov, M. Y. Shverdin, B. Rusnak, A. M. Tremaine, F. V. Hartemann, C. W. Siders, D. P. Mcnabb, C. P. J. Barty, "Characterization and applications of a tunable, laser-based, MeVclass Compton-scattering gamma-ray source,” Phys. Rev. ST-AB, 13 (2010) 070704.

[4] O. Teşileanu, D. Ursescu, R. Dabu and N. V. Zamfir, "Extreme Light Infrastructure - Nuclear Physics," Journal of Physics: Conference Series 420 (2013) 012157.

[5] R. Hajima, N. Kikuzawa, N. Nishimori, T. Hayakawa, T. Shizuma, K. Kawase, M. Kando, E. Minehara, H. Toyokawa, H. Ohgaki, "Detection of radioactive isotopes by using laser Compton scattered gamma-ray beams," Nuclear Instruments and Methods in Physics Research A 608, (2009) 557.

[6] http://asta.fnal.gov/files/ASTA_Proposal_October_2013.pdf

[7] https://portal.slac.stanford.edu/sites/ard_public/facet/Documents/FACET-II\%20Proposal\%20v6.pdf

[8] http://www.bnl.gov/atf/

[9] H. Schwoerer, B. Liesfeld, H.-P. Schlenvoigt, K.-U. Amthor, and R. Sauerbrey, "Thomson-Backscattered X Rays From Laser-Accelerated Electrons," Phys. Rev. Letters, 96 (2006) 014802.

[10] K. Ta Phuoc, S. Corde, C. Thaury, V. Malka, A. Tafzi, J. P. Goddet, R. C. Shah, S. Sebban, A. Rousse, "All-optical Compton gamma-ray source," Nature Photonics 6 (2012) 308.

[11] S. Chen, N. D. Powers, I. Ghebregziabher, C. M. Maharjan, C. Liu, G. Golovin, S. Banerjee, J. Zhang, N. Cunningham, A. Moorti, S. Clarke, S. Pozzi, D. P. Umstadter, "MeV-Energy X Rays from Inverse Compton Scattering with Laser-Wakefield Accelerated Electrons," Phys. Rev. Lett. 110, (2013) 155003.

[12] G. Sarri, D. $\square$ J. Corvan, W. Schumaker, J. $\square$ M. Cole, A. Di Piazza, H. Ahmed, C. Harvey, C. $\square$ H. Keitel, K. Krushelnick, S. $\square$ P. $\square$ D. Mangles, Z. Najmudin, D. Symes, A. $\square$ G. $\square$ R. Thomas, M. Yeung, Z. Zhao, and M. Zepf, "Ultra-high brilliance multi-MeV $\gamma$-ray beams from nonlinear relativistic Thomson scattering," Phys. Rev. Lett 113 (2014) 224801.

[13] B.J. Quiter S. G. Prussin, B. Pohl, J. Hall, J. Trebes, G. Stone, M.-A. Descalle, "A method for high-resolution x-ray imaging of intermodal cargo containers for fissionable materials," J. Appl. Phys. 103, 064910 (2008).

[14] M. Johnson, J. M. Hall, D. P. McNabb, M. J. Tuffley, M. W. Ahmed, S. Stave, "Using Quasi-Monoenergetic Photon Sources To Probe Photo-Fission Resonances," AIP Conference Proceedings, Vol. 1336 Issue 1, (2011) 590.

[15] J. Pruet, D. P. McNabb, C. A. Hagmann, F. V. Hartemann, C. P. J. Barty, "Detecting clandestine material with nuclear resonance fluorescence," Journal Of Applied Physics 99, 123102 (2006).

[16] W. P. Leemans, R. W. Schoenlein, P. Volfbeyn, A. H. Chin, T. E. Glover, P. Balling, M. Zolotorev, K. J. Kim, S. Chattopadhyay, and C. V. Shank, "X-Ray Based Subpicosecond Electron Bunch Characterization Using 90 Thomson Scattering," Phys. Rev. Letters 77 (1996) 4182.

[17] A. Jochmann, A. Irman, M. Bussmann, J. P. Couperus, T. E. Cowan, A. D. Debus, M. Kuntzsch, K. W. D. Ledingham, U. Lehnert, R. Sauerbrey, H. P. Schlenvoigt, D. Seipt, Th. Stöhlker, D. B. Thorn, S. Trotsenko, A. Wagner, and U. Schramm, "High Resolution EnergyAngle Correlation Measurement of Hard X Rays from Laser-Thomson Backscattering,"Phys. Rev. Letters, 111, (2013) 114803.

[18] S.G. Rykovanov, C. G. R. Geddes, J.-L. Vay, C. B. Schroeder, E. Esarey, W. P. Leemans, "Quasi-monoenergetic femtosecond photon sources from Thomson Scattering using laser plasma accelerators and plasma channels," J. Physics B 47 (2014) 234013

[19] http://science.energy.gov/ /media/hep/pdf/accelerator-rd-stewardship/Lasers_for_Accelerators_Report_Final.pdf

[20] C. B. Schroeder, E. Esarey, C. Geddes, C. Benedetti, and W. P. Leemans, "Physics considerations for laser-plasma linear colliders," PRSTAB 13, (2010) 1010301.

[21] E. Esarey, C. B. Schroeder, and W. P. Leemans, "Physics of laser-driven plasma-based electron accelerators," Rev. Modern Phys. 81 (2009) 1229.

[22] E. Cormier-Michel, V. $\square$ H. Ranjbar, D. $\square$ L. Bruhwiler, J. $\square$ R. Cary, M. Chen, C. $\square$ G. $\square$ R. Geddes, G. $\square$ R. Plateau, N. $\square$ H. Matlis, and W. $\square$ P. Leemans, "Design principles for high quality electron beams via colliding pulses in laser plasma accelerators," Phys. Rev. ST-AB 17 (2014) 091301.

[23] C.G.R. Geddes, Cs. Toth, J. van Tilborg, E. Esarey, C.B. Schroeder, D. Bruhwiler, C. Nieter, J. Cary, W.P. Leemans, "High-quality electron beams from a laser wakefield accelerator using plasma-channel guiding," Nature, V 438, pp. 538-41 (2004); Faure et al, ibid. Mangles et al, ibid.

[24] W.P. Leemans, B. Nagler, A.J. Gonsalves, Cs. Toth, K. Nakamura,3, C.G.R. Geddes, E. Esarey, C.B. Schroeder, S.M. Hooker, "GeV electron beams from a centimetre-scale accelerator," Nature Physics, 2, (2006) 696.

[25] N. H. Matlis, C. G. R. Geddes, G. R. Plateau, E. Esarey, C. Schroeder, D. Bruhwiler, E. Cormier-Michel, M. Chen, L. Yu, and W. P. Leemans, "Controlling electron injection in laser plasma accelerators using multiple pulses," Proc. Advanced Accelerator Concepts Workshop, AIP 1507 (2012) 717. 
[26] E. Esarey, R. F. Hubbard, W. P. Leemans, A. Ting, and P. Sprangle, "Electron Injection into Plasma Wakefields by Colliding Laser Pulses," Phys. Rev. Letters 79 (1997) 2682.

[27] J. Faure, C. Rechatin, A. Norlin, A. Lifschitz, Y. Glinec and V. Malka, "Controlled injection and acceleration of electrons in plasma wakefields by colliding laser pulses, 'Nature 444 (2006) 737-739.

[28] C.G.R. Geddes, N.H. Matlis, S. Steinke, E. Esarey, K. Nakamura, G.R. Plateau, C.B. Schroeder, Cs. Toth, W.P. Leemans, "High energy, low energy spread electron bunches produced via colliding pulse injection," Proc. Adv. Accel. Concepts Workshop (2014).

[29] G. R. Plateau, C. G. R. Geddes, D. B. Thorn, M. Chen, C. Benedetti, E. Esarey, A.J. Gonsalves, N.H. Matlis, K. Nakamura, C.B. Schroeder, S. Shiraishi, T. Sokollik, J. van Tilborg, Cs. Toth, S. Trotsenko, T.S. Kim, M. Battaglia, Th. Stoehlker, and W. P. Leemans, "Low-Emittance Electron Bunches from a Laser-Plasma Accelerator Measured using Single-Shot X-Ray Spectroscopy,” Phys. Rev. Letters 109 (2012) 064802.

[30] W. $\square$ P. Leemans, A. $\square$ J. Gonsalves, H.-S. Mao, K. Nakamura, C. Benedetti, C. $\square$ B. Schroeder, Cs. Tóth, J. Daniels, D. $\square$ E. Mittelberger, S. $\square$ S. Bulanov, J.-L. Vay, C. $\square$ G. $\square$ R. Geddes, and E. Esarey, "Multi-GeV electron beams from capillary-discharge-guided sub-petawatt laser pulses in the self-trapping regime," Phys. Rev. Lett. 113 (2014) 245002.

[31] C. Lin, J. van Tilborg, K. Nakamura, A. J. Gonsalves, N. H. Matlis, T. Sokollik, S. Shiraishi, J. Osterhoff, C. Benedetti, C. B. Schroeder, Cs. Tóth, E. Esarey, and W. P. Leemans, "Long-Range Persistence of Femtosecond Modulations on Laser-Plasma-Accelerated Electron Beams," Phys. Rev. Letters" 108, (2012) 094801.

[32] C.G.R. Geddes, Cs. Toth, J. van Tilborg, E. Esarey, C.B. Schroeder, J. Cary, W.P. Leemans, "Guiding of Relativistic Laser Pulses by Preformed Plasma Channels," Phys. Rev. Letters, 95, 14, 145002/1-4 (2005).

[33] C. B. Schroeder, E. Esarey, C. Benedetti, W. P. Leemans, "Control of focusing forces and emittances in plasma-based accelerators using near-hollow plasma channels,” Phys. Plasmas 20, (2013) 080701.

[34] I. Ghebregziabher, B.A. Shadwick, D. Umstadter, "Spectral bandwidth reduction of Thomson scattered light by pulse chirping," Phys. Rev. ST-AB 16 (2013) 030705.

[35] S.G. Rykovanov, C.G.R. Geddes, C.B. Schroeder, E. Esarey, W.P. Leemans, "Controlling the spectral shape of nonlinear Thomson scattering with proper laser chirping," arXiv:1412.2517 and submitted to Phys. Rev. ST-AB. 